\title{
Transform-Invariant Recognition by Association in a Recurrent Network
}

\author{
Néstor Parga \\ Edmund Rolls \\ Oxford University, Department of Experimental Psychology, Oxford OX1 3UD, \\ England
}

Objects can be reconized independently of the view they present, of their position on the retina, or their scale. It has been suggested that one basic mechanism that makes this possible is a memory effect, or a trace, that allows associations to be made between consecutive views of one object. In this work, we explore the possibility that this memory trace is provided by the sustained activity of neurons in layers of the visual pathway produced by an extensive recurrent connectivity. We describe a model that contains this high recurrent connectivity and synaptic efficacies built with contributions from associations between pairs of views that is simple enough to be treated analytically. The main result is that there is a change of behavior as the strength of the association between views of the same object, relative to the association within each view of an object, increases. When its value is small, sustained activity in the network is produced by the views themselves. As it increases above a threshold value, the network always reaches a particular state (which represents the object) independent of the particular view that was seen as a stimulus. In this regime, the network can still store an extensive number of objects, each defined by a finite (although it can be large) number of views.

\section{Introduction}

Single neurons with responses that are relatively invariant with respect to, for example, the position, size, and even view of an object or face are present in the primate temporal visual cortical areas (see, e.g., Gross, Desimone, Albright, \& Schwartz, 1985; Tanaka, Saito, Fukada, \& Moriya, 1990; Rolls, 1984, 1992, 1994, 1995, 1996b; Rolls, Booth, \& Treves, 1996). How could such invariant representations be formed? One suggestion is that there is a short-term memory trace built into the learning rule implemented in the visual system, which enables, for example, successive views of the same object to be associated together (Foldiak, 1991; Rolls, 1992, 1994, 1995, 1996b). Because the statistics with which objects are normally viewed in the visual world result in different aspects (e.g., views) of the same object being seen close together in time, such a learning rule might enable different views of 
objects to be associated together. In contrast, views of different objects only rarely, on average, occur close in time. Thus, a neuron could learn by a simple form of Hebbian associativity coupled with a short-term memory trace to respond to any views of an object but to no views of other objects. Foldiak (1991) showed that translation-invariance learning over a one-dimensional input array was possible for a simple winner-take-all network with a decaying trace of previous neuronal activity and an associative Hebb rule. Rolls (1992, 1994, 1995) and (Rolls \& Treves, 1997) suggest that invariant representations could be formed in the visual system for two-dimensional images using a multistage architecture, with convergence onto a neuron at any one layer from a small region of the preceding layer, a short-term trace of preceding neuronal activity, soft competition between the neurons to produce distributed representations, and a Hebb-like learning rule. In the theory as suggested, the trace was implemented by a short-term trace in the postsynaptic neuron, and this enabled neurons to learn which inputs from the preceding stage tended to occur close together in time.

Possible neurophysiological mechanisms suggested for the trace included the continuing firing of single neurons in the visual system, which often lasts for $300 \mathrm{~ms}$ following a $20 \mathrm{~ms}$ presentation of a stimulus (Rolls \& Tovee, 1994; Rolls, Tovee, Purcell, Stewart, \& Azzopardi, 1994), which could be implemented by recurrent collateral connections between pyramidal cells in the same layer or in adjacent cortical stages in the cortico-cortical hierarchy of stages in the visual system (see Rolls, 1992, 1994, 1995); the rather slow unbinding of glutamate from the NMDA receptors after they have been activated (this may be seen after even $100 \mathrm{~ms}$ ); and slow changes intracellularly induced after the $\mathrm{Ca}^{2+}$ entry, which is one step in the induction of long-term potentiation. Wallis, Rolls, \& Foldiak (1993) and Wallis and Rolls (1997) produced a simulation, VisNet, of this theory of the formation of invariant representations in the visual system proposed by Rolls (1992, 1994, 1995, 1996a, 1996b). The simulation showed that translation, size, and view invariance could be learned by such a network.

In developing these ideas further, we turn to an approach that allows an analytic formalism to be brought to bear on the issue of the storage capacity of a recurrent network, which performs, for example, view-invariant recognition of objects by associating together different views of the same object that tend to occur close together in time. The architecture with which the invariance is computed is a little different from that already described. In the model of Rolls $(1992,1994,1996 b ;$ Wallis \& Rolls, 1997), the postsynaptic memory trace enabled different afferents from the preceding stage to modify their synapses onto the same postsynaptic neuron (see Figure 1). In that model there were no recurrent connections between the neurons, although such connections were one way in which it was postulated the memory trace might be implemented, by simply keeping the representation of one view or aspect active until the next view appeared. Then an association would occur 


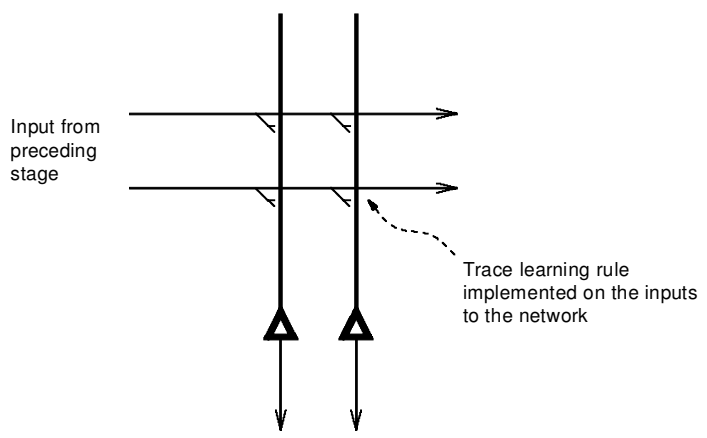

Figure 1: A trace learning rule is implemented in the feedforward inputs to a nonrecurrent network.

between representations that were active close together in time (within, e.g., 100-300 ms).

In our model, there is a set of inputs with fixed synaptic weights to a network. The network itself is a recurrent network. For the purposes of this article, we are concerned primarily with how the recurrent network would operate once the synaptic matrix has been formed, not with how the synaptic matrix is formed. In section 4, we will describe a set of neuronal operations that could lead to our synaptic matrix. Let us say for the moment that in the case of a recurrent network, we expect that the trace rule is implemented on the recurrent collaterals (see Figure 2).

In the context of recurrent networks, we can consider two main approaches. First, a very simple approach to store in a synaptic weight matrix the $s$ views of an object. This consists of equally associating all the views to each other, including the association of each view with itself (that is, the diagonal terms of the association matrix). Choosing in Figure 3 an example such that objects are defined in terms of five different views, this might produce (if each view produced firing of one neuron at a rate of 1) a block of $5 \times 5$ pairs of views, contributing to the synaptic efficacies each with value 1 . Object 2 might produce another block of synapses of value 1 further along the diagonal and symmetric about it. Each object or memory could then be thought of as a single attractor with a distributed representation involving five elements (each element representing a different view). Then the capacity of the system in terms of the number $P_{o}$ of objects that can be stored is the number of separate attractors that can be stored in the network. For random fully distributed patterns, this is as shown numerically by Hopfield (1982),

$$
P_{o}=0.14 C,
$$

where there are $C$ inputs per neuron (and $N=C$ neurons if the network is fully connected). The synaptic matrix envisaged here does not consist of 


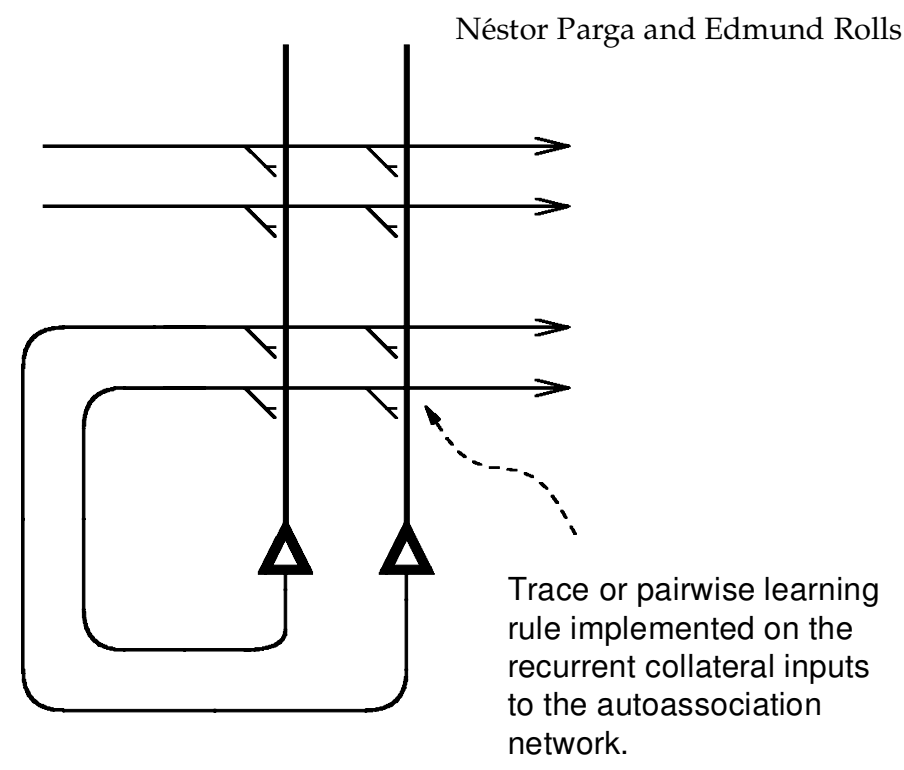

Figure 2: A trace or pairwise associative learning rule is implemented in the recurrent collateral synapses of an autoassociative memory.

random fully distributed binary elements; instead we will assume that it has a sparseness $w=\frac{s}{N} n$, where $s$ is the number of views stored for each object, from any of which the whole representation of the object must be reconized. ( $n$ is the average number of active neurons in the element, or portion of the pattern, that describes one view.) In this case, one can show (as in Gardner, 1988; Tsodyks \& Feigel'man, 1988) that the number of objects,

$$
P_{o}=\frac{k C}{w \ln (1 / w)}
$$

where $C$ is the number of synapses on each neuron devoted to the recurrent collaterals from other neurons in the network, and $k$ is a factor of order one that depends weakly on the detailed structure of the rate distribution, the connectivity pattern, and so forth. A problem with this proposal is that as the number of views per object increases to a large number (e.g., > 20), the network will fail to retrieve correctly the internal representation of the object starting from any one view (which is only a fraction $1 / s$ of the length of the stored pattern that represents an object). This is because if the cue given by one of the views becomes too small, the internal representation of the stimulus will fall outside the attraction basin of the attractor associated to the object.

The second approach, which we discuss in detail here, is to consider the operation of the network when the associations between pairs of views can be described by a matrix that has the general form shown in Figure 4. Such 


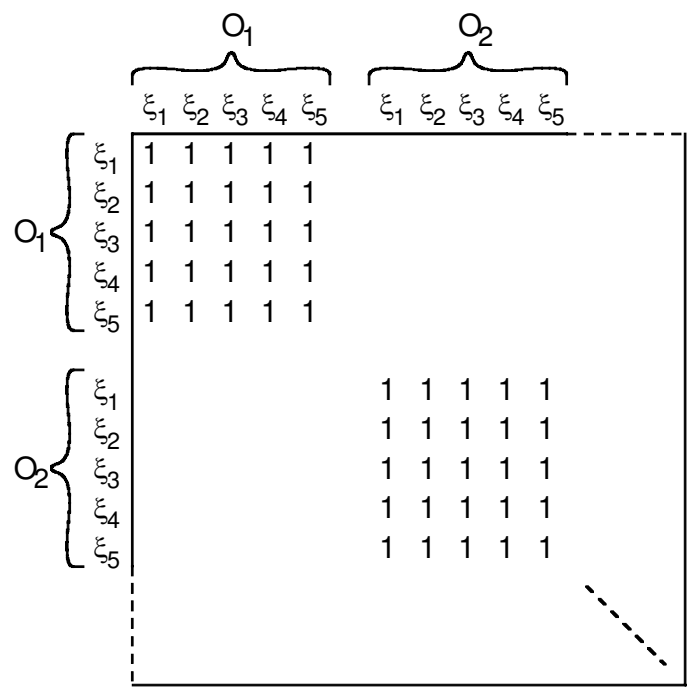

Figure 3: A schematic illustration of the first type of associations contributing to the synaptic matrix considered (see the text).

an association matrix might be produced by different views of an object appearing after a given view with equal probability, and synaptic modification occurring of the view with itself (giving rise to the diagonal term), and of any one view with that which immediately follows it. ${ }^{1}$ The same matrix might be produced not only by pairwise association of successive views because the association rule allows for associations over a short time scale of, say, 100 to $200 \mathrm{~ms}$, but might also be produced if the synaptic trace had an exponentially decaying form over several hundred milliseconds, allowing associations with decaying strength between views separated by one or more intervening views. The existence of a regime, for values of the coupling parameter between pairs of views in a finite interval, such that the presentation of any of the views of one object leads to the same attractor regardless of the particular view chosen as a cue, is the main issue that we deal with here. A related problem we also deal with is the issue of the capacity of this type of synaptic matrix: How many objects can be stored and retrieved correctly in a view-invariant way? As we will show in the model presented here, their number grows linearly with the number of neurons.

${ }^{1}$ Strictly speaking the matrices in Figures 3 and 4 do not refer to the same thing. In the first case, it denotes the matrix of synaptic efficacies; in the second, it is the association between two patterns (the matrix $X$ in equation 2.1). 


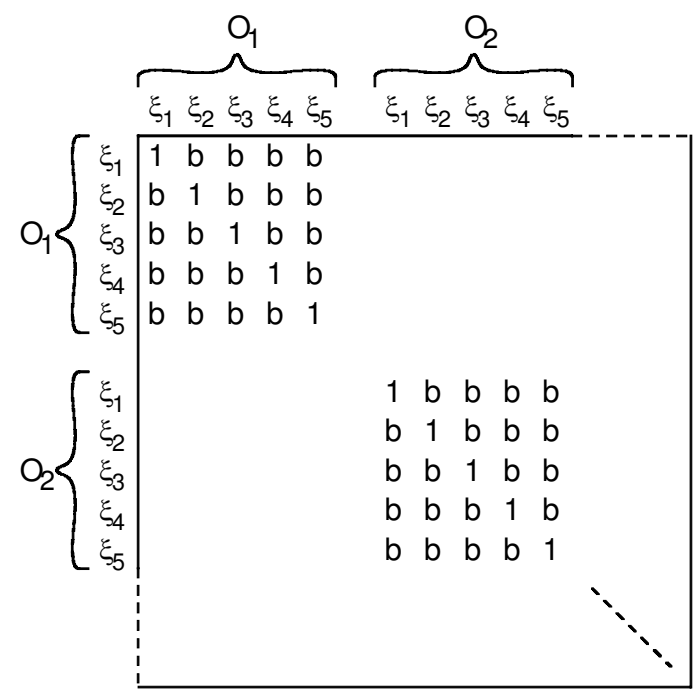

Figure 4: A schematic illustration of the second and main type of synaptic matrix considered (see the text).

Some of the groundwork for this approach was laid by the work of Amit and collaborators (Griniasty, Tsodyks, \& Amit, 1993).

A variant of the second approach is to consider that the remaining entries in the matrix shown in Figure 4 all have a small value. This would be produced by the fact that sometimes a view of one object would be followed by a view of a different object, when, for example, a large saccade was made, with no explicit resetting of the trace. On average, any one object would follow another rarely, and so the case is considered when all the remaining associations between pairs of views have a low value.

\section{The Model}

We will consider a simplemodel to see if it can store a large number of objects in a view-invariant way. In order to have a problem solvable by standard statistical physics techniques (Amit,1988), we will make several simplifying hypothesis. The $N$ neurons in the model are binary objects. Denoting the state of neuron $i$ by $S_{i}$, this variable can take only the values +1 and -1 .

The views of the objects will be chosen as $P$ special states of the network. Different views, even if they belong to the same object, will be taken as uncorrelated random variables. The interpretation of these states is that they are the internal representations in some internal layer of stimuli that might differ in the way an object is presented: different illumination or color, 
different view or scale. In this internal layer, all these possibilities appear as uncorrelated states of the network, and the only relation between these states when they represent the same object is that they will be associated with each other. We will refer to all of these internal states as views, regardless of the real difference in the stimuli they represent. We will also assume that the network is fully connected, and we will take a coding rate (or sparseness) $w=0.5$.

By convention we will label the views, placing first all those associated with, say, object 1 , then all the views defining object 2 , and so on. We will denote the internal representation of the $\mathcal{v}$ th view by $\xi^{\nu}$, and the state of neuron $j$ in this pattern by $\xi_{j}^{\nu}(j=1, \ldots, N)$ (see Figure 4$)$. For simplicity, we will assume that all the objects are defined by the same number $s$ of views; in this way, the patterns labeled by $v=1, \ldots, s$ refer to views of the first object, and so forth. The variables $\xi_{j}^{v}$ will be selected to be +1 or -1 , with probability $w=0.5$.

All pairs of views of the same object are coupled to each other. We will denote the coupling between the patterns $\xi^{\mu}$ and $\xi^{\nu}$ as $X_{\mu \nu}$. Since we are not considering the effect of an association between views of different objects, this matrix is made of blocks of size $s \times s$-one block for each of the $P_{o}$ objects.

These considerations lead us to propose the following form of the synaptic matrix,

$$
J_{i j}=\frac{1}{2 N} \sum_{\mu=1}^{s P_{o}} \xi_{i}^{\mu} X_{\mu \nu} \xi_{j}^{\nu},
$$

which, more explicitly, contains a contribution from association between views of the form

$$
\frac{1}{N} \sum_{\mu \neq \nu}^{s P_{o}} b_{\mu \nu} \xi_{i}^{\mu} \xi_{j}^{\nu}
$$

where $b_{\mu \nu}$ (the off-diagonal elements of $X_{\mu \nu}$ ) is the strength of the association between views $\xi^{\mu}$ and $\xi^{\nu}$. The value of the coupling of one view with itself will be taken equal to one. In the case where all pairs of different views of a given object are equally correlated with strength $b_{\mu \nu}=b$ for all $\mu \neq \nu$, while views of different objects are not correlated, the matrix $X$ takes the block form shown in Figure 4 for $s=5$. This is the case we will deal with, and the block matrix of size $s \times s$ will be denoted by $\mathcal{O}^{(s)}$. It is natural to think that $b$ is smaller than the strength of the association of one view with itself, that is, $b<1 .^{2}$ For an arbitrary value of the sparseness the synaptic matrix

2 The choice of equal strength for all the pairs simplifies the numerical analysis; the equations in this section can be easily extended to the general case. 
given in equation 2.1 is not a good choice. One should use, for example, the one studied by Tsodyks and Feigel'man (1988). An analysis of the capacity properties for other values of $w$ requires finding the explicit solution of the model for the new synaptic matrix.

Our purpose is to show that this network has an object phase-that is, a phase where each object is represented by a different attractor of the recurrent network. If all views of the same object are contained in its associated attraction basin, then view-invariant recognition of the object will be achieved when either one of the views or a pattern sufficiently close to one of them is presented as a stimulus.

If each object were defined by a single view, this model reduces to the Hopfield model, which can store a number of patterns linear in N. More precisely, in this saturation regime, the number of stored objects grows as $\alpha N$, where $\alpha$ has to be less than $\alpha_{c}=0.14$. In this case, the view is very close to the attractor itself. If this model is modified to contain a finite number of more complex objects (those having several views) in the background of those simple one-view objects, it is reasonable to expect that the complex objects will still define stable states if the coupling between the views is strong enough. The case where all the $P_{o} \simeq O(N)$ objects are defined by a finite number of views requires a more careful analysis. This can be done with tools from statistical physics that were used some time ago to solve the standard Hopfield model (Amit, Gutfreund, \& Sompolinsky 1985; Amit 1988).

Before presenting the result of this calculation, let us see how to characterize the behavior of the network for given values of the parameters $\alpha$ and $b$. The useful quantities for this purpose are the overlaps of the state of the network $\left\{S_{i}\right\}(i=1, \ldots, N)$ with the views of one of the objects $(\mu=1, \ldots, s)$ :

$$
m_{\mu}=\frac{1}{N} \sum_{i=1}^{N} S_{i} \xi_{i}^{\mu} .
$$

If the network is presented with a stimulus close to one of the views, say $\xi^{\mu_{0}}$, then all these overlaps initially will be very small, except $m_{\mu_{0}}$, which will be close to 1.0. Over time, these overlaps will change until a fixed point is reached. If the final state is similar to the initial one, then we will say that the network is in the view phase. But it could also happen that the stable state has overlaps with more than one view. When it has similar overlaps with all the patterns representing the $s$ views of the object seen initially, we will say that this point is in the view-invariant object phase, or simply in the object phase. Because of the symmetry between the views, no matter which view is taken as the initial condition of the network, it will always reach the same attractor. Another possibility is that the system will get to a state where all the overlaps are null. Then there is neither view nor object retrieval, and the system is beyond its capacity. The region where this happens is called, by analogy with a similar situation in statistical physics models, a spin- 
glass phase. For the simple model we are proposing here, there are no more complex solutions because of the symmetry of the synaptic matrix.

Let us continue by defining the free energy of a system with Hamiltonian,

$$
H=\sum_{i \neq j}^{N} J_{i j} S_{i} S_{j} .
$$

The free energy is then computed as:

$$
f=-\frac{1}{\beta} \lim _{N \rightarrow \infty} \frac{1}{N}\left\langle\left\langle\log \operatorname{Tr}_{S} \exp [-\beta H]\right\rangle\right\rangle_{\xi}
$$

where the angular brackets denote the average over the variables $\xi$ and the symbol $T r_{S}$ refers to a sum over all possible states of the network. An extra parameter, $\beta$, has been introduced that acts as the inverse of a temperature. At the end, however, we will consider only the zero temperature limit. This means that the spike emission dynamics is given simply by

$$
S_{i}(t+1)=\operatorname{sign}\left[\sum_{j \neq i}^{N} J_{i j} S_{j}(t)\right] .
$$

In the thermodynamic description given by the free energy $f$, the state of the system is described in terms of macroscopic quantities. This means that instead of the overlaps defined in equation 2.3, where the microstate $\left\{S_{i}\right\}_{i=1, \ldots, N}$ is used, one should use their thermal averages. These are computed by averaging over all the microstates weighed with the Boltzmann distribution that appears in equation 2.5. From now on, we will use $m_{\mu}$ to denote these overlaps. More explicitly, denoting the thermal average with single angular brackets, we have

$$
m_{\mu}=\frac{1}{N} \sum_{i=1}^{N}\left\langle S_{i}\right\rangle \xi_{i}^{\mu}
$$

To check the existence of a transition from the view to the object phase, one has to look for states such that their overlap with all the views of a given object are, in principle, $m_{\mu} \sim O(1)$, while the overlaps with the views of other objects are zero (actually $O(1 / \sqrt{N})$ ). In terms of these macroscopic quantities, the view phase is characterized by $m_{\mu}=0$ for $\mu \neq \mu_{0}$. On the other hand, in the object phase, these $s$ overlaps are nonzero and equal. It turns out that these order parameters are not enough to describe the behavior of the network properly. This is because there are $O(N)$ overlaps $O(1 / \sqrt{N})$ with the views of the other objects. This effect is taken into account 
by an order parameter, $r$, which gives the mean square overlap of all the views of the other objects:

$$
r=\frac{1}{\alpha} \sum_{\mu \nu>s}\left\langle\left\langle m_{\mu}\left(X^{2}\right)_{\mu \nu} m_{\nu}\right\rangle\right\rangle_{\xi} .
$$

Finally one also has to consider the possibility that the $m_{\mu}$ 's are zero, but the order parameter defined as

$$
q=\frac{1}{N}\left\langle\sum_{i=1}^{N}\left\langle S_{i}\right\rangle^{2}\right\rangle_{\xi}
$$

is not zero.

Another relevant point is that the mean-field solution of this model is exact. This is because the network is fully connected. Then the order parameters satisfy a set of mean-field, self-consistent equations. In order to find them, one first has to compute and express the free energy in terms of the order parameters and then extremize it with respect to them.

The evaluation of the free energy of this kind of system is now standard (Mezard, Parisi, \& Virasoro, 1987). The free energy and the mean-field equations have been obtained for a general coupling matrix $X$ (although with a different motivation) by Cugliandolo and Tsodyks (1994). (See appendix I of their work for details of the calculation.) Here we describe just the main steps of the algebra and present the results the free energy and for the meanfield equations of our problem (that is, for the case where $X$ decomposes in $P_{o} s \times s$ blocks as in Figure 4).

Very briefly, the computation proceeds as follows: One first uses the representation of the logarithm

$$
\log z=\lim _{n \rightarrow 0} \frac{z^{n}-1}{n}
$$

in equation 2.5. Then each of the $n$ factors in $f$ is identified with a "replica" of the network. Notice that all of them share the same set of internal representations of the views. At this point the quenched average over these variables can be done easily. Now the free energy can be written in terms of integrals over the order parameters $q, r$, and $m_{\mu}$ (only the $O(1)$ overlaps, that is, $\mu \leq s$ ). The final step is to make the ansatz that the problem is symmetric under permutations of the $n$ replicas. Then the remaining integrals can be readily solved in the large $N$ limit (keeping $\alpha$ fixed) by the saddle point method.

After some lengthy but straightforward algebra, one obtains that the free energy at zero "temperature" (i.e., for $\beta^{-1}=0$ ) is given by:

$$
f=\frac{1}{2}\left(\alpha+\sum_{\gamma, \lambda=1}^{s} m_{\gamma} \mathcal{O}_{\gamma \lambda}^{(s)} m_{\lambda}+\alpha r c+J(q)-2\left\langle\left\langle\sigma \operatorname{erf}\left(\frac{\sigma}{\sqrt{2 \alpha r}}\right)\right\rangle\right\rangle_{\xi}\right.
$$




$$
\left.\left.-\sqrt{\frac{\alpha r}{2 \pi}} /\left\langle\exp \left(-\frac{\sigma^{2}}{2 \alpha r}\right)\right\rangle\right\rangle_{\xi}\right)
$$

Here $\mathcal{O}_{\gamma \lambda}^{(s)}$ is the matrix element $(\gamma, \lambda)$ of $\mathcal{O}^{(s)} ; \operatorname{erf}(x)$ denotes the error function, and $c$ is $(1-q) \beta$ in the large $\beta$ limit. The angular brackets indicate an average over the views. The function $J(q)$ is given by

$$
J(q)=-\frac{\alpha}{s}\left[\frac{1-b+s b}{1-c(1-b+s b)}+(s-1) \frac{1-b}{1-c(1-b)}\right],
$$

and $\sigma$ is defined as

$$
\sigma=\sum_{\gamma \lambda}^{s} m_{\gamma} \mathcal{O}_{\gamma \lambda}^{(s)} \xi^{\lambda}
$$

For given values of the load parameter $\alpha$ and the strength of the association between a pair of views of the same object $b$, the values of the overlaps of the stationary state of the network with the representation of the $s$ views of the object are given by the solution of the following equations:

$$
\begin{aligned}
& m_{\nu}=\left\langle\left\langle\xi_{\nu} \operatorname{erf}\left(\frac{\sum_{\gamma \lambda}^{s} m_{\gamma} \mathcal{O}_{\gamma \lambda}^{(s)} \xi^{\lambda}}{\sqrt{2 \alpha r}}\right)\right\rangle\right\rangle_{\xi} \\
& c=\sqrt{\frac{2}{\pi \alpha r}}\left\langle\left\langle\exp \left[-\left(\frac{\sum_{\gamma \lambda}^{s} m_{\gamma} \mathcal{O}_{\gamma \lambda}^{(s)} \xi^{\lambda}}{\sqrt{2 \alpha r}}\right)\right)^{2}\right]\right\rangle_{\xi} \\
& r=\frac{1}{s}\left[\frac{1-b+s b}{1-c(1-b+s b)}\right]^{2}+\frac{(s-1)}{s}\left[\frac{1-b}{1-c(1-b)}\right]^{2} .
\end{aligned}
$$

These equations describe the retrieval properties of one object (let's say object $\mathcal{A}_{0}$ ) immersed in a background of another $P_{o}=\alpha_{0} N$ objects (here $\alpha_{0}=\alpha / s$ is the load parameter relevant for objects); the effect of these other objects is contained in the parameter $r$ (this is computed trivially from the last equation once $c$ is known), which gives the mean square overlap of all the views of the other objects. The network might also have more complicated states, say, a state given by a mixture of two objects. These states are not described by the equations above; however, they are not relevant if the cue is sufficiently close to one of the views of object $\mathcal{A}_{0}$.

\section{Results}

The simplest way to look for solutions of these equations is to use an iterative procedure; for instance, for the $s$ overlaps $m_{\mu}$, we have (the time $t$ denotes 
Table 1: Capacity (Critical Value of the Load Parameter $\alpha=\alpha_{0} s$ ) of the Object Phase for Several Values of the Number of Views $s$.

\begin{tabular}{|rl|}
\hline \multicolumn{1}{|c|}{$s$} & $\alpha_{0} s$ \\
\hline 3 & 0.087 \\
5 & 0.081 \\
7 & 0.077 \\
9 & 0.076 \\
11 & 0.073 \\
\hline
\end{tabular}

the iteration number):

$$
m_{\mu}(t+1)=\left\langle\left\langle\xi_{\mu} \operatorname{erf}\left(\frac{\sum_{\gamma \lambda}^{s} m_{\gamma}(t) \mathcal{O}_{\gamma \lambda}^{(s)} \xi^{\lambda}}{\sqrt{2 \alpha r}}\right)\right\rangle\right\rangle_{\xi} .
$$

As an initial condition, the overlaps are taken $m_{\mu}=0$, except one of them, for example, $m_{\mu_{0}}$, which is given a value close to one. The iterations should proceed until a fixed point of this dynamics is reached. However, we have noticed that this naive method does not work, it either fails to reach a fixed point or if it does find one, one cannot be sure of its stability. For this reason, we have preferred to work directly with the equations that result from performing a steepest descent of the free energy. The method is briefly described in the appendix.

The solution of equations 2.13 through 2.15 shows that the object phase is indeed present in a region of the parameter space defined by $\alpha$ and $b$. As expected, it appears when the coupling between views $b$ is strong enough to destabilize the view phase and $\alpha$ is small enough to prevent the noise coming from the storage of an $O(N)$ number of objects from spoiling the retrieval of one of them. For a fixed value of $b$, one can compute the value of $\alpha$ where the transition from the object to the spin-glass phase takes place. The result is shown in Table 1 for $b=0.80$ for several values of $s$, up to 11 views per object. Note that the capacity of the network decreases slowly as $s$ increases.

The complete phase diagram of the model is presented in Figure 5 for a fixed number of views $(s=5)$. The view phase where the views themselves define attractors appears at the left lower corner of the figure; these states disappear at large $\alpha$ when the network makes the usual transition to the spin-glass phase and at large $b$ where the system reaches the object phase. The capacity in terms of the number of objects that can be stored in the network is given by the value of the almost horizontal line in Figure 5 divided by $s$.

It is interesting to note that the object phase exists even for $b$ smaller than the values shown in this figure. It is present even for $b=0$. The standard Hopfield model at low values of $1 / \beta$ already has a set of stable symmetric phases where the state of the system has a uniform overlap with an odd number of patterns. In that case, however, they are not relevant because 


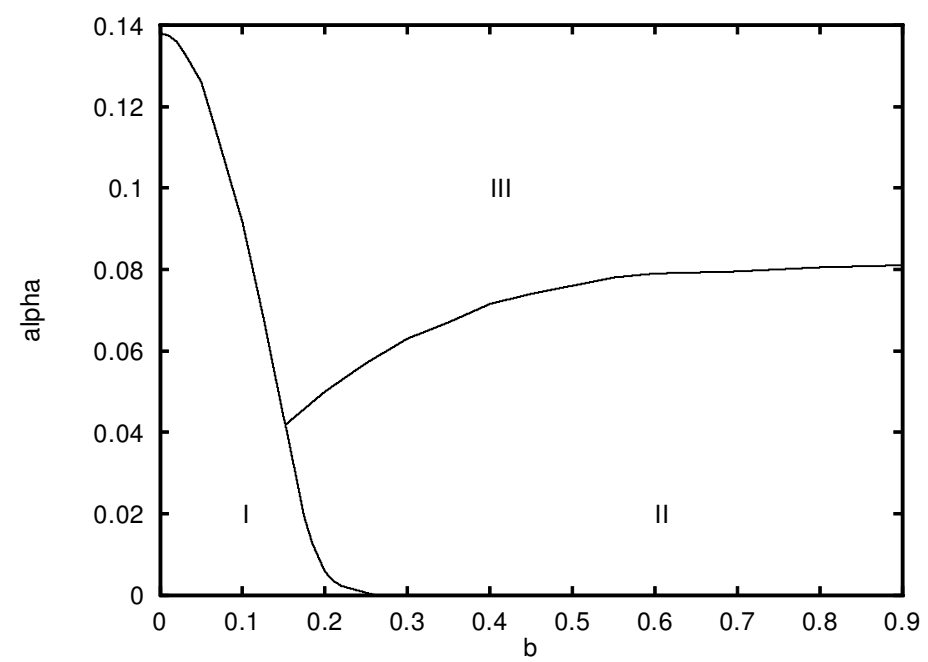

Figure 5: The phase diagram of the model for $s=5$. Region I is the standard view phase, where the views themselves are retrieved correctly. In this region, objects are not stored in a view-invariant fashion. Region II is the phase where objects are stored as attractors; any stimulus close to some of the views will elicit the same response. Finally in region III, the spin-glass phase, no storage of information is possible.

one is interested in the retrieval of the patterns (which we here call the views), and the symmetric states do not show up when the stimulus is close to one of them. As $b$ is increased, it will reach a value where one pattern cannot support an attractor by itself and then the symmetric (object) states will emerge, giving rise to the (object) phase we were looking for. One consequence of this scenario is that the model can support only objects defined by an odd number of views; however, one expects this to be the result of the artificial aspects of the model. In fact, as is discussed in Amit et al. (1985), the properties of these solutions depend on the distribution of the patterns $\xi_{j}^{\nu}$. In a more realistic model, with the state of neurons defined in terms of their firing rates, we expect this restriction to disappear.

\section{Discussion}

In this work, we have shown that invariant object recognition is feasible in attractor neural networks. The system is able to store and retrieve in a view-invariant way an extensive number of objects, each defined by a finite set of views. What is implied by "extensive" is that the number of objects is proportional to the size of the network. The crucial factor that defines 
this size is the number of connections per neuron. In the case of the fully connected networks considered in this article, the size is thus proportional to the number of neurons. To be more specific, the number of objects that can be stored is $0.081 \mathrm{~N} / 5$, when there are 5 views of each object. The number of objects is $0.073 \mathrm{~N} / 11$, when there are 11 views of each object. This is an interesting result in network terms, in that $s$ views, each represented by an independent random set of active neurons, can, in the network described, be present on the same "object" attraction basis. It is also an interesting result in neurophysiological terms; the number of objects that can be represented in this network scales linearly with the number of recurrent connections per neuron.

Although the explicit numerical calculation was done for a rather small number of views per object (up to 11), the basic result that the network can support this kind of phase is expected to hold for any number of them (the only requirement being that it does not increase with the number of neurons). This is, of course, enough. Once an object is defined by a set of views, when the network is presented with a somewhat different stimulus, or an interpolation of some of the views, or a noisy version of one of them, it will still be in the attraction basin of the object attractor.

Some of the assumptions taken to simplify the problem are not relevant in what regards the existence of this regime. This is the case of the assumption that all pairs are coupled to each other with the same strength. Choosing them with a certain distribution will only change the details of where the object phase appears.

Here we have been mainly concerned with the retrieval properties of the network. Because of that, we started our analysis from a synaptic matrix where the associations between the views had already been learned. One can consider, however, how this matrix is built through a learning procedure that takes place as one object is seen from different perspectives or under different conditions. Learning could proceed as follows: since a fixed view is normally seen for longer times than transitions to another view, it is natural to assume that associations of one view with itself will be built up first, while those between different views appear later. This means that the association between views will happen in the presence of attractors for each view. This is possible because at this stage of learning, the strength of the association between pairs is still small, and, as we have shown, the network will sit in a view phase where the attractors are defined by the views themselves. Under these conditions, when the stimulus corresponds to, say, view $v_{1}$, the network will reach its attractor and will maintain sustained activity for about $300 \mathrm{~ms}$ (Rolls \& Tovee, 1994). But within this time scale, the object can already present a different view $v_{2}$ as the input stimulus. For a short period of time, the neuronal firing may be in a state that reflects both $v_{1}$ and $v_{2}$. During this time, the appropriate synaptic modification could take place. After these two views are seen consecutively several times, the contribution made by this particular process to the synaptic efficacies will be higher than 
the threshold required to make a transition to the object phase, and views will start to be recognized as a single object. For a different learning protocol, this mechanism has been studied in some detail by Amit and Brunel (1995) and Brunel (1996).

The model emphazises the role of the attractors to implement the association between views, but other neurophysiological mechanisms could also participate to reinforce the association. The unbinding of glutamate by the NMDA receptors lasting for about 100 ms could produce this effect. Also the trace rule used in Foldiak (1991) and Wallis and Rolls (1997) is just one possible way to describe any of these memory effects mathematically.

A study of invariant object representations using recurrent networks has also been done by O'Reilly and Johnson (1994). Although this is interesting work, the analysis is mainly numerical and the capacity properties of the model are not fully understood. Invariant representation of faces in the context of attractor neural networks has been discussed by Bartlett and Sejnowski (1996) in terms of a model where different views of faces are presented in a fixed sequence (Griniasty et al. 1993). This is not, however, the general situation; normally any pair of views can be seen consecutively, and they will become associated. The most general version of the model presented in this work contemplates this possibility. ${ }^{3}$ The synaptic matrix in equation 2.1 refers to this situation, although later we found it more convenient to study the simpler case, where all the views are coupled to each other with the same strength.

We wish to note the different nature of the invariant object recognition problem studied here and the paired-associate learning task studied in Miyashita and Chang (1988), Miyashita (1988), and Sakai and Miyashita (1991). In the invariant object recognition case, no particular learning protocol is required to produce an activity of the inferotemporal cells responsible for invariant object recognition maintained for $300 \mathrm{~ms}$. The learning can occur rapidly, and the learning takes place between stimuli (e.g., different views) that occur with no intervening delay. In the paired-associate task, the monkeys must learn to associate together two stimuli that are separated in time (by a number of seconds), and this type of learning can take weeks. During the delay period, the sustained activity is rather low in the experiments, and thus the representation of the first stimulus that remains is weak and can be associated with the second stimulus only poorly. Formally, however, the learning mechanism could be treated in the same way as we have used here for invariant object recognition. The experimental difference is that in the paired-associate task used by Miyashita and Chang (1988), it is the weak memory of the first stimulus that is associated with the second

${ }^{3}$ After this work was submitted, we found that in a more recent publication, Bartlett and Sejnowski (1997) relaxed the condition on the presentation of the views in a fixed sequence. In this respect, their recurrent synaptic matrix is closer to ours. 
stimulus. In contrast, in the invariance learning, it would be the firing activity being produced by the first stimulus (not the weak memory of the first stimulus) that can be associated together.

The mechanisms described here would apply most naturally when a small number of representations need to be associated together to represent an object. One example is associating together what is noted when an object is seen from different perspectives. Another example is scale, with respect to which neurons early in the visual system tolerate scale changes of approximately 1.5 octaves, so that the whole scale range could be covered by associating together a limited number of such representations (see Rolls, 1994, 1996b). The mechanism would not be so suitable when a large number of different instances would need to be associated together to form an invariant representation of objects, as might be needed for translation invariance. For the latter, we propose a solution in a multilayer network, with a local solution being implemented at each stage (Rolls, 1994, 1995; Wallis \& Rolls, 1996). We have envisaged the local solution for translation invariance at each stage as being performed by a trace rule implemented between the inputs to a stage and the postsynaptic neurons in a stage. However, both types of mechanism, implemented in the feedforward connections or in the recurrent collateral connections, could contribute (separately or together) to achieving invariant representations. Part of the interest of the approach described in this article is that it allows analytic investigation, and this is what we have introduced here.

\section{Appendix}

Here we explain how equations 2.13 through 2.15 were solved. A straightforward way to perform a steepest descent on the free energy given in equation 2.10 is to update the order parameters according to the variation of the free energy with respect to $m_{\mu}, r$, and $c$. The complication of this approach is that it yields $P+2$ coupled equations, even when we know that $r$ and $c$ are trivially related at the fixed point, as equation 2.15 shows. One can make convergence faster by imposing this relation as a constraint on the dynamics by means of a Lagrange multiplier. The algebra is rather simple, and one finally finds the following equations for the updating of $m_{\mu}, r$, and $c$ :

$$
\begin{aligned}
\delta m_{v}= & -\eta(1-a)\left[m_{v}-\left\langle\xi^{v} \operatorname{erf}\left(\frac{\sigma}{\sqrt{2 \alpha r}}\right)\right\rangle_{\xi}\right] \\
& +a\left[M-\left\langle Y_{\xi} \operatorname{erf}\left(\frac{\sigma}{\sqrt{2 \alpha r}}\right)\right\rangle_{\xi}\right] \\
\delta r= & -\eta \frac{\alpha}{2}\left[c-\sqrt{\frac{2}{\pi \alpha r}}\left\langle\exp \left(-\frac{\sigma^{2}}{2 \alpha r}\right)\right\rangle_{\xi}\right]+\eta \lambda
\end{aligned}
$$




$$
\delta c=-\eta \frac{\alpha}{2}[r-K(c)]-\eta \lambda \frac{\delta K(c)}{\delta c} .
$$

In these equations we have defined:

$$
\begin{aligned}
& M=\sum_{\lambda=1}^{s} m_{\lambda} \\
& Y_{\xi}=\sum_{\lambda=1}^{s} \xi^{\lambda} \\
& K(c)=\frac{1}{s}\left[\frac{1-a+s a}{1-c(1-a+s a)}\right]^{2}+\frac{(s-1)}{s}\left[\frac{1-a}{1-c(1-a)}\right]^{2},
\end{aligned}
$$

and $\lambda$ is chosen in such a way that if $r=K(c)$ holds at a given time (in particular at $t=0$ ) then it also holds at the next time step:

$$
r+\delta r=K(c+\delta c) .
$$

Although it is correct to solve equations A.1 through A.3 to see if the invariant-recognition phase is reached upon presentation of one view, the connection between these equations and the dynamics defined by equation 2.6 is subtle. Equation 2.6 refers to the evolution of a microscopic state. It decreases systematically the value of the Hamiltonian, equation 2.4. On the other hand, equations A.1 through A.3 decrease the free energy. Strictly speaking, the fixed point is not the same. This is because once a number $O(N)$ of views have been stored in the synaptic matrix, there will be many metastable states with a large (i.e., close to one) overlap with a given stored view. Equations A.1 through A.3 reach a fixed point that describes the average properties of these states highly correlated to the stored views. Equation 2.6 reaches one of these states. If the initial condition is modified (but still starting from a state close to one of the views), the final state will probably be a different one, although it will still have a large overlap with the stored pattern. A study of the number of metastable states in an associative memory network has been done by Gardner (1986).

\section{Acknowledgments}

This research was supported by the Medical Research Council, PG8513790, by an E. U. Human Capital and Mobility grant CHRX-CT92-0063, and by a Spanish grant PB96-47.

\section{References}

Amit, D. (1988). Modeling brain function. Cambridge: Cambridge University Press. 
Amit, D., \& Brunel, N. (1995). Learning internal representations in an attractor neural network with analogue neurons. NETWORK, 6, 359-388.

Amit, D., Gutfreund, H., \& Sompolinsky, H. (1985). Spin-glass models of neural networks. Phys. Rev., A 32, 1007-1018.

Bartlett, M. S., \& Sejnowski, T. J. (1996). Learning viewpoint invariant representations of faces in an attractor network. Communication presented at the 18th Cognitive Science Meeting, San Diego, CA.

Bartlett, M. S., \& Sejnowski, T. J. (1997). Viewpoint invariant face recognition using independent component analysis and attractor networks. In M. Mozer, M. Jordan, \& T. Petsche (Eds.), Advances in neural information processing systems 9, Cambridge, MA: MIT Press.

Brunel, N. (1996). Hebbian learning of context in recurrent neural networks. Neural Computation, 8, 1677.

Cugliandolo, L. F., \& Tsodyks, M. V. (1994). Capacity of networks with corrrelated attractors. Journal of Phsyics A, 27, 741-755.

Foldiak, P. (1991). Learning invariance from transformation sequences. Neural Computation, 3, 193-199.

Gardner, E. (1986). Structure of metastable states in the Hopfield model. Journal Physics, A 19, L1047-L1052.

Gardner, E. (1988). The space of interactions in neural network models. Journal Physics, A 21, 257-270.

Griniasty, M., Tsodyks, M. V., \& Amit, D. J. (1993). Conversion of temporal correlations between stimuli to spatial correlations between attractors. Neural Computation, 5, 1.

Gross, C. G., Desimone, R., Albright, T. D., \& Schwartz, E. L. (1985). Inferior temporal cortex and pattern recognition. Exp. Brain Res. Suppl., 11, 179-201.

Hopfield, J. J. (182). Neural networks and physical systems with emergent collective computational abilities. Natl. Acad. Sci. USA, 79, 2554-2558.

Mezard, M., Parisi, G., \& Virasoro, M. A. (1987). Spin glass theory and beyond. Singapore: World Scientific.

Miyashita, Y. (1988). Neuronal correlate of visual associative long-term memory in the primate temporal cortex. Nature, 335, 817-820.

Miyashita, Y., \& Chang, H. S. (1988). Neuronal correlate of pictorial short-term memory in the primate temporal cortex. Nature, 331, 68-70.

O'Reilly, R. C., \& Johnson, M. H. (1994). Object recognition and sensitive periods: A computational analysis of visual imprinting. Neural Computation, 6, 357389.

Rolls, E. T. (1984).Neurons in the cortex of the temporal lobe and in the amygdata of the monkey with responses selective for faces. Human Neurobiology, 3, 209222.

Rolls, E. T. (1992). Neurophysiological mechanisms underlying face processing within and beyond the temporal cortical visual areas. Phil. Trans. Roy. Soc., 335, 11-21.

Rolls, E. T. (1994). Brain mechanisms for invariant visual recognition and learning. Behavioural Processes, 33, 1134-138.

Rolls, E. T. (1995). Learning mechanisms in the temporal lobe visual cortex. Behavioural Brain Res., 66, 177-185. 
Rolls, E. T. (1996a). Roles of long term potentiation and long term depression in neural network operations in the brain. In M. S. Fazeli \& G. L. Collingridge (Eds.), Cortical plasticity: LTP and LTD. Oxford: Bios.

Rolls, E. T. (1996b). A neurophysiological and computational approach to the functions of the temporal lobe cortical visual areas in invariant object recognition. In L. Harris \& M. Jenkin (Eds)., Computational biological mechanisms of visual coding. Cambridge: Cambridge University Press.

Rolls, E. T., Booth, M. C. A., \& Treves, A. (1996). View-invariant representations of objects in the inferior temporal visual cortex. Society for Neuroscience Abstracts, 22.

Rolls, E. T., \& Tovee, M. J. (1994). Processing speed in the cerebral cortex, and the neurophysiology of visual backward masking. Proc. Roy. Soc., B 257, 9-15.

Rolls, E. T., Tovee, M. J., Purcell, D. G., Stewart, A. L., \& Azzopardi, P. (1994). The responses of neurons in the temporal cortex of primates, and face identification and detection. Exp. Brain Res., 101, 474-484.

Rolls, E. T., \& Treves, A. (1997). Neuronal networks and brain funciton. Oxford: Oxford University Press.

Sakai, K., \& Miyashita, Y. (1991). Neural organisation for the long-term memory of paired associates. Nature, 354, 152.

Tanaka, K., Saito, C., Fukada, Y., \& Moriya, M. (1990). Integration of form, texture, and color information in the inferotemporal cortex of the Macaque. In E. Iwai \& M. Mishkin (Eds.), Vision, memory and the temporal lobe (pp. 101-109). New York: Elsevier.

Tsodyks, M. V., \& Feigel'man, M. V. (1988). The enhanced storage capacity in neural networks with low activity level. Europhysics Lett., 6, 101-105.

Wallis, G., \& Rolls, E. T. (1997). Invariant face and object recognition in the visual system. Progress in Neurobiology, 51, 167-194.

Wallis, G., Rolls, E. T., \& Foldiak, P. (1993). Learning invariant responses to the natural transformations of objects. In International Joint Conference on Neural Networks (Vol. 2, pp. 1087-1090).

Received February 4, 1997; accepted February 12, 1998. 